\title{
2D Set Partitioning In Hierarchical Tree (SPIHT) On Embedded Devices For Multilead ECG Signal Compression
}

\author{
Grafika Jati*, M. Anwar Ma'sum*, and Wisnu Jatmiko*
}

\begin{abstract}
Heart disease is the number one cause of death in the world according WHO data. One of the mayor cause is the lack of cardiologists. One of the proposed solution is tele-electrocardiograph (Tele-ECG) system. However, the size of ECG data recorded from patient is not small. Therefore, it must be compressed during transmission process. This paper presents development of 2D SPIHT on embedded devices for multilead ECG signal compression. There are two main processes, preprocessing and compression process. Preprocessing includes base wander removal, beat detection, and beat normalization. Compression is started with 2D ECG array construction. Afterward, we apply wavelet decomposition to transform ECG signals from the time domain to frequency domain. Essentially, the outcome of wavelet decomposition has a similar derivative among subbands. This similarity is coded using the Set Partitioning In Hierarchical Tree (SPIHT) algorithm to reduce size of the data. The compression system is successfully implemented in an embedded device: BeagleboardC5. In this research, we use 8 leads (Lead-1, Lead-2, and Lead-V1 to V6) ECG signals taken from Physionet database. Experiment results show that the percentage root-mean-square difference (PRD) of this system is of $\mathbf{1 . 4 7 6 3}$ for the compression ratio of 8.

Keyword: ECG Signals Compression, SPIHT, Embedded Devices, Beagleboard
\end{abstract}

\section{INTRODUCTION}

Heart disease is the number one cause of death in the world. Data from the World Health Organization in 2008 indicate that each year nearly 17 million people die from heart disease or Cardiovascular disease (CVD). Moreover, the World Health Organization predicts that by 2030, 23 million people die from heart disease each year ${ }^{1}$ Heart disease is a term for various kinds of errors caused by cardiac disfunction. One example is Arithmia heart disease. It is a heart rhythm disorder or disturbance, i.e. erratic and or irregular (can happen too fast or too slow) heart rate.

The risk of heart disease can be reduced by regular controls on the performance of the heart. Cardiac performance will be analyzed by medical personnel to get proper treatment. However, there are difficulties in providing adequate treatment, for example, due to the lack of cardiologist. According to Perki, Cardiovascular Specialist Doctors Association of Indonesia, there are 459 cardiologist for 240 million people in Indonesia 2 It shows that a doctor handles about 522876 people. The patiet-to-cardiologist ratio is very small. In addition, they are not evenly distributed in each

\footnotetext{
*) Faculty of Computer Science, University of Indonesia. grafika.jatiegmail.com

1 http://who.int/en/

2 http://inaheart.org.
}

province in Indonesia. Thus, it is crucial to build a system that records heart distance or Tele-Eelectrocardiograph.

Tele-Electrocardiography signal recording technology is based on electrocardiogram (ECG) and communications technology. The recording needs to produce an accurate ECG. Therefore, we need recording from multilead ECG in a few days continuously; making the data quite large. The data are transmitted over communication networks in realtime. The amount of data sent affects the delivery process. Hence, at first. we need to process the ECG signal data. The process is applied to the ECG signals in order to obtain a smaller data size with high accuracy. The transmision process becomes an important concern since the overall process must be done quickly.

There are several research related to compression of ECG signals. Sani et al propose ECG Signal compression using SPIHT with beat reordering for optimization [1]. Linnenbank et al use Huffman Code for ECG signal compression [5]. Lu et al propose 1D SPIHT compression for single and multi-lead signals [3]. Pooyan et al use wavelet compression for ECG signals with 1d SPIHT [2]. Goudarzi et al propose an ECG compression algorithm based on two dimensional multiwavelet transforms [4].

In this paper, we propose an embedded system that can be used as tele-electrocardiograph device. The system consists of several devices that have roles ranging from receiving the ECG signal, compressing the data signal to displaying the results of signal compression. We utilize the the 2D SPIHT algorithm for signal compression. The proposed embedded system is developed in a compact form so can be used in high mobility. This system also has an user-friendly GUI (Grapichal User Interface).

\section{SYSTEM ARCHITECTURE}

\section{A. Software}

The compression program is developed using $\mathrm{C}$ and $\mathrm{C}++$ programming language. There is also a graphical user intterface (GUI) to facilitate the user. The system displays the results of signal compression and bit-stream of signal.

Signal compression results are shown in figure 2. Figure 3 shows the results of signal compression in the form of a bit stream. The user interface consists of several panels with the following functions:

- to display the signal

- to select the leads of the signal to be displayed

- to run or pause the compression process

- to display the bit-stream signal 


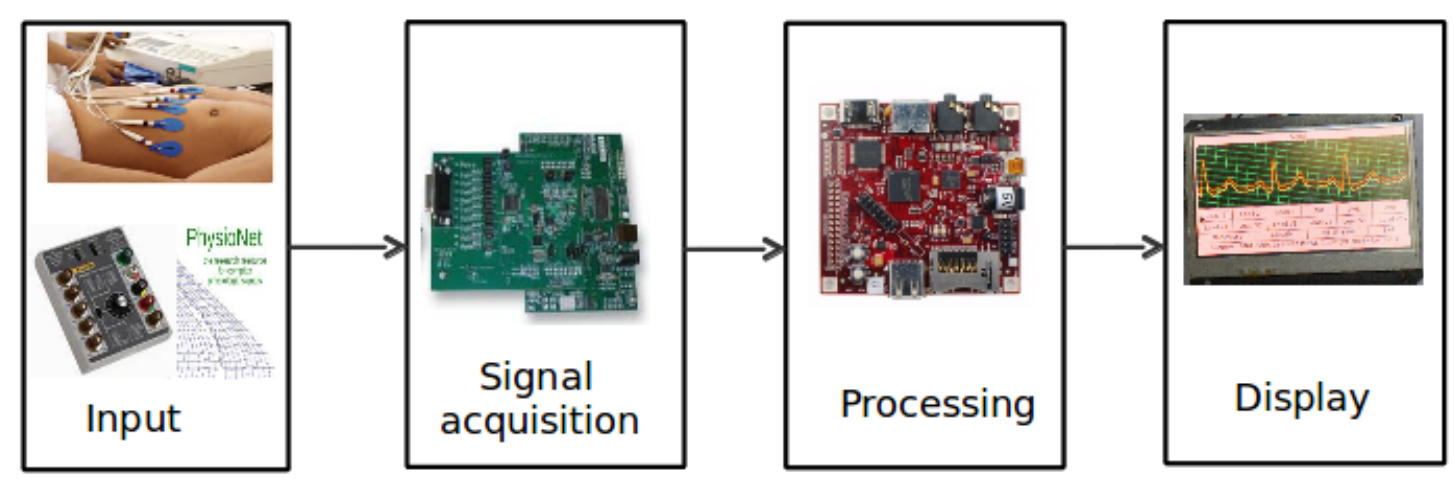

Fig. 1. The Hardware Architecture.

- to classify the signals

- to exit the program

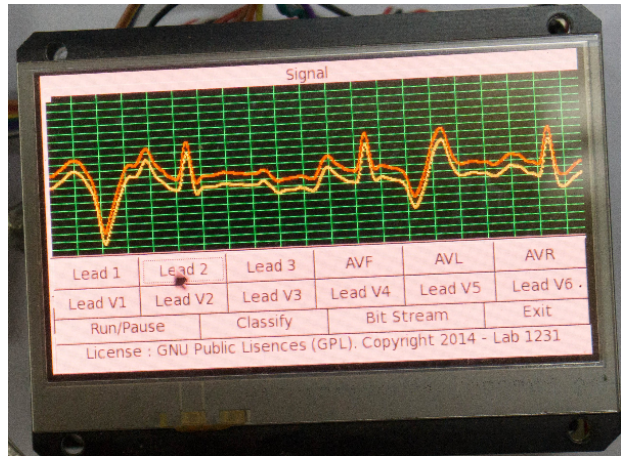

Fig. 2. The reconstructed signals User Interface; the red signal is the original signal, while the yellow signal is the reconstructed signal.

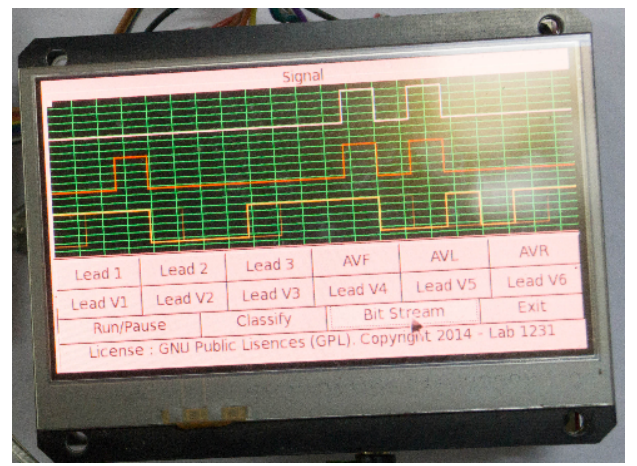

Fig. 3. The Bit-stream User Interface; there are three bit-streams of the three ECG leads. Each bit-stream is shown in different colors such as white, red, and yellow. Each bit-stream $b \in\{0,1\}$, where a value of 0 and 1 indicates low and high bits, respectively.

\section{B. Hardware}

The hardware architecture consists of three parts: signal acquisition unit, processing unit, and display unit. It is shown in figure 1. The signal acquisition unit is used to capture ECG signal from patient and to convert the signal into digital signals. We use an ADS1296 chip as the signal acquisition unit. This chips has a analog-to-digital converter that meets the standards of Advancement of Medical Instrumentation (AAMI) and the International Electronic Commission (IEC). This chip measures the ECG signal from a patient up to 12 leads. This chip has a sampling rate of $250 \mathrm{~Hz}$ up to $32 \mathrm{KHz}$ and 24-bit resolution.

The main parts of this system is a processing unit that serves to process multilead ECG signals. We utilize an ARM Cortex-A8 OMAP3530 module contained in a beagle board 3 The module executes a command in $1 \mathrm{GHz}$ with $512 \mathrm{MB}$ of memory. The module is also equipped with coprocessor DSO TMS320C64X for signal processing. We employ the Unix operating system to develop the system.

We use BeagleTouch for the display unit. The BeagleTouch has $480 \times 272$ resolution with a $262 \mathrm{~K}$ color touchscreen OLED display. Since it has a touch screen, the BeagleTouch can also be used to interact with the system and provide user input. To provide power to system, we used BeagleJuice. BeagleJuice is capable of delivering a $1.5 \mathrm{~A}$ current for 6.5 hours. In addition, it accomodates a direct plug from househould electric network through a AC-DC adapter.

\section{2D SPIHT COMPRESSION}

Figure 4 depicts the 2D SPIHT workflow system (figure 4) for data compression electrocardiogram (ECG). The system consists of two subsystem, namely: preprocessing and main subsystems. The preprocessing subsystem deals with an early processing stage for preparing the signal. Whereas, the main subsytem performs the main stage of compression. Eventually, the system outputs bit-streams, whose size is smaller than that of the original input signal. Notice that the size of the bit-stream depends on the compression ratio used in 2D SPIHT coding. An example of the bit-stream is $\{128,2,7,1,0,0,0,1,1,0,1,0\}$.

\section{A. Preprocessing}

The preprocessing consists of three stages:

- Base Wonder Removal

This stage eliminates the deviation (drift) from baseline ECG signal. This yields a clean signal.

http://beagleboard.org/ 


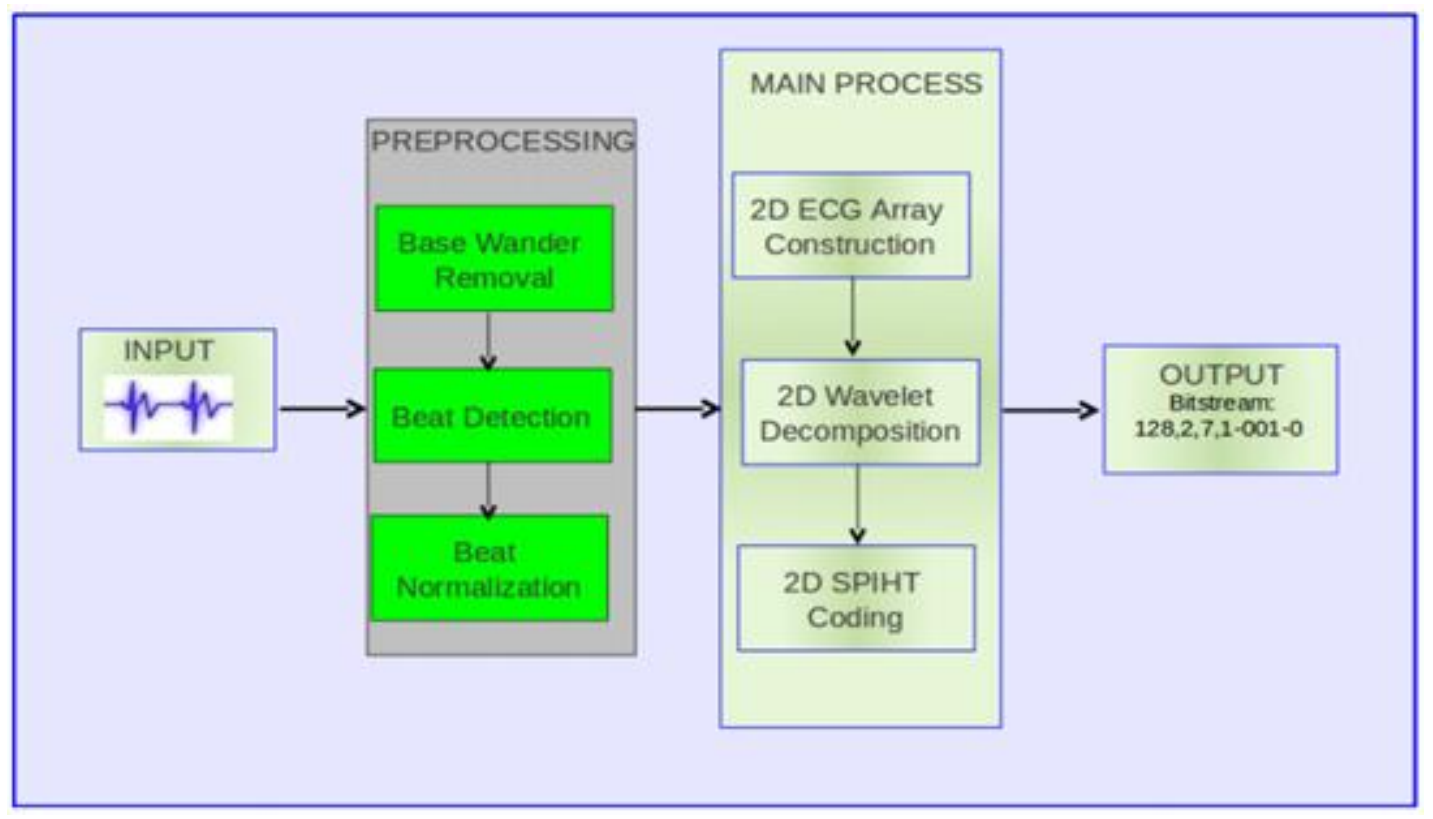

Fig. 4. The System Workflow.

- Beat Detection

This stage determines the beat of clean signals.

- Beat Normalization

This stage standardizes the amount of sample for each beat. In our system, each beat has 128 samples.

\section{B. 2D ECG Array Construction}

The 2D Array Construction phase forms two-dimensional arrays of arrangement throughout each beat in the ECG signals. This stage aims to obtain the regularity of the ECG signal. According Mamaghanian [6], an ECG signal has pseudo-periodic signals so that each heartbeat cycle always repeats the same pattern, namely P-QRS-T. A generated 2D array (matrix) has a high degree of regularity because every row matrix consists of a vector representing one cycle of the ECG signal. A 2D array has 128 rows, in which each row has 128 samples. Thus, the matrix size is of $128 \times 128$.

\section{C. $2 D$ Wavelet Decomposition}

The 2D Wavelet Decomposition generates wavelet decomposition of the 2D array that has been formed. Implementation of Wavelet Decomposition using Library 1D/2D Wavelet Transform artificial Rafat Hussain, MIT Licens 4 . We use the bior4.4 mother wavelet. The highest decomposition level that can be achieved is level 7 because the matrix size is of $128 \times 128$, where $128=2^{7}$. The resulting wavelet coefficients become the input of the 2D SPIHT compression process.

\section{D. $2 D$ SPIHT}

The Set Partitioning in Hierarchical Trees (SPIHT) is a compression method that was developed from the EZW (Embedded Zero Tree) method [7]. The SPIHT coding is an wavelet-based technique. It has a sort of encoder charge

4 http://wavelet 2 d. sourceforge.net/ wavelet transform coefficients using the partitioning algorithm based on the sub-band of the pyramid is formed. Decoder then uses signal received to reconstruct the wavelet coefficients of the inverse transform is then performed to obtain the original signal. SPIHT requires no training and can produce embedded bitstream, yet still can maintain signal quality.

Wavelet decomposition, in principle, has the same derivative between subbands. The similarity of the offspring is eventually encoded using SPIHT compression algorithm. SPIHT assumes that the structure of the wavelet decomposition as a tree structure called spatial orientation tree. This structure has a spatial relationship on the hierarchical pyramid. Figure 5 shows the spatial orientation tree is represented in the pyramid structure. Each node in the tree is a wavelet coefficient of the ECG signal is indicated by the coordinates of the location where the two fields dimensions.

SPIHT consists of three main stages

1) Setting up the set of coordinates of wavelet coefficients based on spatial orientation tree

The following set of coordinates used in SPIHT:

- $H$ : set of coordinates of all root in spatial orientation tree.

- $O(i, j)$ : set of all offspring of a node $(i, j)$

- $D(i, j)$ : set of all descendant of node $(i, j)$

- $L(i, j): D(i, j)-O(i, j)$

Set of offspring $O(i, j)$ from node $(i, j)$ can be obtained by formula:

$$
O(i, j)=(2 i, 2 j),(2 i, 2 j+1),(2 i+1,2 j),(2 i+1,2 j+1)
$$

2) Sorting Pass

This stage is used to sort the coefficients based on the 


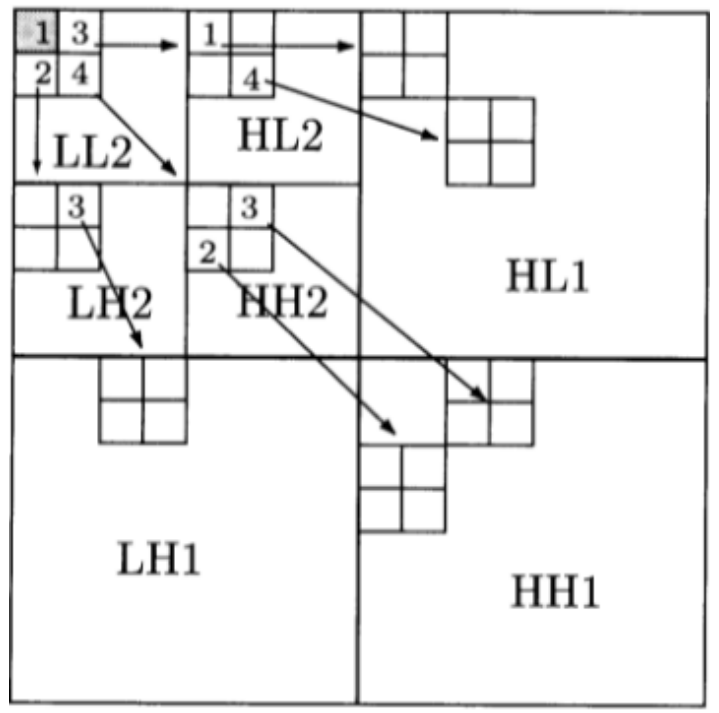

Fig. 5. The Spatial Orientation Tree

value of the coefficient. The sorting use threshold that is updated on every process of sorting and refinement. SPIHT requires grouping the coefficients in the three sets, namely:

a) LIP (list of insignificant pixel) is the coordinates of insignificant coefficients based on the current threshold.

b) LSP (list of significant pixel) is the coordinates of significant coefficients based on the current threshold.

c) LIS (list of insignificant sets) is a set of subtress roots coordinates which are insignificant.

LIS sets of coefficients is updated and if the coefficient becomes significant then the coordinates of the coefficient is moved from LIP to LSP. This produces a bit stream organized progressively. Set in the LIS is evaluated later. If significant set is found, then the set will be broken down and removed from the LIS. A new subset that has more than one element is added back to the LIS. Each subset that contains a coefficient is evaluated whether significant or not. If significant, the subset is added to the LSP, otherwise, it will be added to the LIP.

3) Refinement pass

Each of LSP is checked. If it is significant, a one is sent and its magnitude reduced by the current threshold. Otherwise, a zero is sent.

\section{EXPERIMENT AND RESULT}

\section{A. Dataset}

We use the multilead St. Petersburg electrocardiogram (ECG) data from Physionet ATM. One patient data stored in one record signals that are already equipped with annotations. One record consists of 12 lead signals, but in this research only used 8 signal leads, namely Lead I, Lead II, Lead V1, Lead V2, V3 Lead, Lead V4, V5 Lead, Lead V6.
Lead is an 8-lead the main leads in the 12 lead ECG. The signal on each record has a resolution of 12 bits. This means that one sample point signal is represented in 12 bits. We use record $\mathrm{I} 01$ is shown in figure 7 .

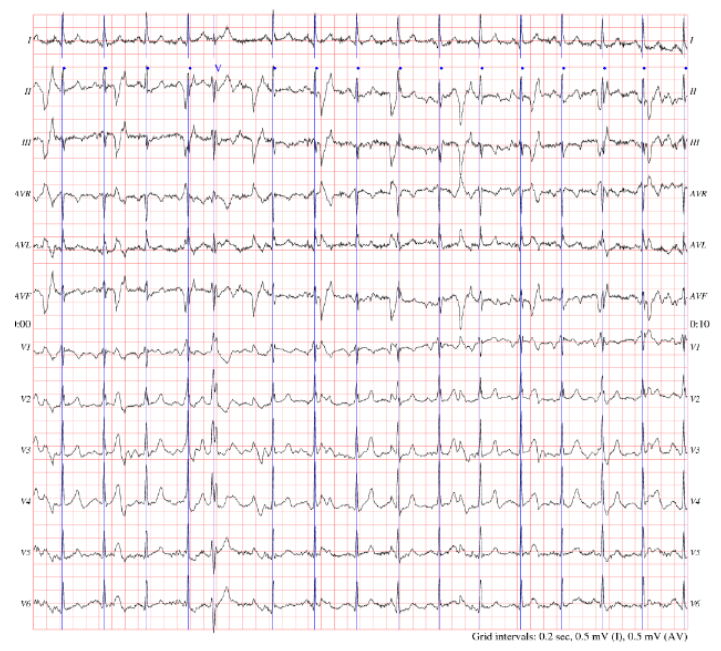

Fig. 7. The Record Signals Sample; courtesy of http://www. physionet.org/

\section{B. Evaluation}

The performance of a compression method is measured by using two parameters: compression ratio(CR) and precentage root-mean-square difference(PRD). Compression ratio is formulated as follows.

$$
C R=\frac{n_{\text {bit_ori }}}{n_{\text {bit_compressed }}}
$$

where $n_{\text {bit_ori }}$ and $n_{\text {bit_compressed }}$ indicate the number of bits in the original signal and the number of bits in the compressed signal, respectively.

The PRD shows the percentage difference between the results of the reconstruction of the original signal with the compressed signal. PRD is measured with the following equation:

$$
P R D=\sqrt{\frac{\sum_{n=1}^{N}(x(n)-\hat{x}(n))^{2}}{\sum_{n=1}^{N} x^{2}(n)}}
$$

where $x$ and $\hat{x}$ indicate the input signal and reconstructed signal, respectively.

Experiments on 2D SPIHT compression method is performed on a computer using Matlab. The proposed method is also performed in an embedded device. One experiment compares the performance of the proposed method between the one that run on the Matlab and that run on a embedded device using the CR and PRD parameters. Table Th phows the results of experiments signal compression using records I01 from the Physionet database.

The table shows that the larger the compression ratio, the larger the obtained PRD. The compression ratio of 8 obtains the smallest PRD of 1.4763 . The compression ratio of 128 (the largest) obtains the PRD of 12.315. Two signals are similiar if their PRD values are zero. 
Lead $i$ th $=1$
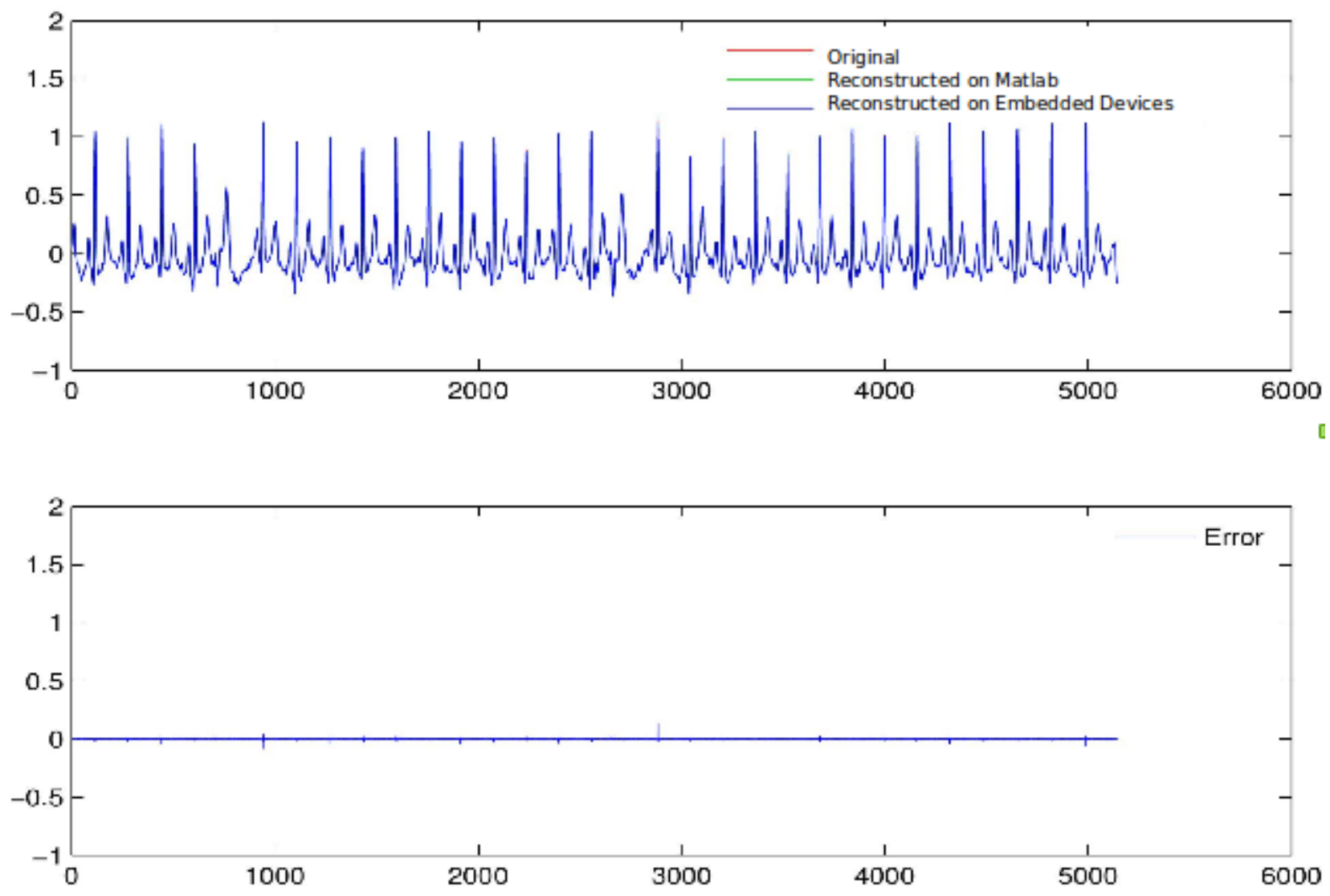

Fig. 6. The Reconstructed Signal Record I01 Lead

TABLE I

Percent of Root Square Difference Comparison Between Running in Matlab and Embedded Devices with Various COMPRESSION RATIO

\begin{tabular}{ccc}
\hline & \multicolumn{2}{c}{ PRD } \\
\cline { 2 - 3 } CR & Matlab & Embedded Device \\
\hline 8 & 1.4763 & 1.4763 \\
16 & 2.1681 & 2.1681 \\
24 & 3.1664 & 3.1664 \\
32 & 4.0608 & 4.0608 \\
64 & 10.998 & 10.998 \\
128 & 12.315 & 12.315 \\
\hline
\end{tabular}

Experiment results show that the compression methods on an embedded device has the same performance with that run on Matlab. This is indicated by PRD values obtained in embedded systems is equal to the value on the computer. It appears that in Figure 6 signal reconstruction almost coincide with the original signal and the right signal coincide with the reconstruction in Matlab.

\section{CONCLUSION}

Heart disease is the number one cause of death in the world according WHO data. One of the mayor cause is the lack of cardiologists. One of the proposed solution is teleelectrocardiograph (Tele-ECG) system. However, the size of ECG data recorded from patient is not small. Therefore, it must be compressed during transmission process. This paper presents development of 2D SPIHT on embedded devices for multilead ECG signal compression. There are two main processes, preprocessing and compression process. Preprocessing includes base wander removal, beat detection, and beat normalization. Compression is started with $2 \mathrm{D}$ ECG array construction. Afterward, we apply wavelet decomposition to transform ECG signals from the time domain to frequency domain. Essentially, the outcome of wavelet decomposition has a similar derivative among subbands. This similarity is coded using the Set Partitioning In Hierarchical Tree (SPIHT) algorithm to reduce size of the data. The compression system is successfully implemented in an embedded device: Beagleboard-C5. In this research, we use 8 leads (Lead-1, Lead-2, and Lead-V1 to V6) ECG signals taken from Physionet database. Experiment results show that the percentage root-mean-square difference (PRD) of this system is of 1.4763 for the compression ratio of 8 . 


\section{ACKNOWLEDGMENT}

This work is supported by Strengthening International Research-Based Collaboration Grant Universitas Indonesia No. 1403/H2.R12/HKP.05.00/Perjanjian/2014

\section{REFERENCES}

[1] Sani M. Isa, Wisnu Jatmiko, Aniati Murni Arymurthy. "Beat Reordering for Optimal Electrocardiogram Signal Compression using SPIHT", in 2012 IEEE International Conference on Systems, Man, and Cybernetics, October 14-17, 2012, COEX, Seoul, Korea

[2] M. Pooyan, A. Taheri, M. Moazami-goudarzi, I. Saboori, and A. Introduction, Wavelet Compression of ECG Signals Using SPIHT Algorithm, vol. 2, no. 3, pp. 212-215, 2005.

[3] Zhitao Lu, Dong Youn Kim, and William A. Pearlman*. "Wavelet Compression of ECG Signals by the Set Partitioning in Hierarchical Trees Algorithm", IEEE TRANSACTIONS ON BIOMEDICAL ENGINEERING, VOL. 47, NO. 7, JULY 2000

[4] M. Moazami-goudarzi, M. H. Moradi, and S. Abbasabadi, High Performance Method for Electrocardiogram Compression Using Two Dimensional Multiwavelet Transform, Computer, no. 1, pp. 1-5, 2005

[5] A. Linnenbank, J. G. C. Kemmelings, S. L. C. Muilwijk, A. Peper, and C. Grimbergen, An effective compression scheme for multilead ecg signals containing ectopie ventricular activation, in Engineering in Medicine and Biology Society, 1992 14th Annual International Conference of the IEEE, vol. 2, 1992, pp. 527528.

[6] Mamaghanian, H., K. N. A. D. V. P. . M. (2011). Compressed sensing for real- time energy-efficient ecg compression on wireless body sensor nodes. IEEE Transac tions on Bio-Medical Engineering.

[7] Said, A. dan Pearlman, W. (1996). A new, fast, and efficient image codec based on set partitioning in hierarchical trees. Circuits and Systems for Video Technology, IEEE Transactions on, 6(3):243250. 